\title{
Molecular diagnosis of eosinophilic meningitis due to Angiostrongylus cantonensis (Nematoda: Metastrongyloidea) by polymerase chain reaction-DNA sequencing of cerebrospinal fluids of patients
}

\author{
Praphathip Eamsobhana ${ }^{1 /+}$, Darawan Wanachiwanawin', \\ Naowarat Dechkum², Anchana Parsartvit ${ }^{3}$, Hoi Sen Yong ${ }^{4}$ \\ 1'Department of Parasitology, Faculty of Medicine Siriraj Hospital ${ }^{2}$ Faculty of Medical Technology, \\ Mahidol University, Bangkok, Thailand ${ }^{3}$ Department of Disease Control, Ministry of Public Health, Nonthaburi, Thailand \\ ${ }^{4}$ Institute of Biological Sciences, University of Malaya, Kuala Lumpur, Malaysia
}

Cerebrospinal fluid (CSF) samples from clinically diagnosed patients with detectable Angiostrongylus cantonensis-specific antibodies $(n=10)$, patients with clinically suspected cases that tested negative for A. cantonensis-antibodies $(n=5)$ and patients with cerebral gnathostomiasis $(n=2)$ and neurocysticercosis $(n=2)$ were examined by a single-step polymerase chain reaction (PCR) method using the AC primers for the 66-kDa native protein gene. The PCR method detected A. cantonensis DNA in CSF samples from four of 10 serologically confirmed angiostrongyliasis cases. The PCR results were negative for the remaining CSF samples. The nucleotide sequences of three positive CSF-PCR samples shared 98.8-99.2\% similarity with the reference sequence of A. cantonensis. These results indicate the potential application of this PCR assay with clinical CSF samples for additional support in the confirmation of eosinophilic meningitis due to A. cantonensis.

Key words: Angiostrongylus cantonensis - eosinophilic meningitis/meningoencephalitis cerebrospinal fluid -PCR - DNA sequencing

\begin{abstract}
Angiostrongylus cantonensis, a metastrongyloid nematode, is the major causative agent of human eosinophilic meningitis or meningoencephalitis in many parts of the world. Humans are infected by ingestion of thirdstage larvae found in molluscan intermediate hosts or other paratenic hosts, such as frogs, freshwater shrimp, crabs or monitor lizards (Eamsobhana \& Tungtrongchitr 2005, Eamsobhana 2006, Cross \& Chen 2007). The infective larvae enter the circulatory system and migrate to the central nervous system (CNS), where they develop into young adults and damage brain tissue, causing intense inflammation. Pathological alterations of the CNS result in abnormal neurological signs and symptoms of meningitis/meningoencephalitis (Wang et al. 2008, Graeff-Teixeira et al. 2009).

A definite parasitological diagnosis is rarely achieved in patients with $A$. cantonensis infection. In the majority of cases, the diagnosis is based on the clinical presentations including severe headache, nuchal stiffness, paraesthesia or visual disturbances. The diagnosis is supported by positive antibody-detection tests for A. cantonensis, e.g., enzyme-linked immunosorbent assay (ELISA) and
\end{abstract}

Financial support: Department of Disease Control, Ministry of Public Health, Thailand

+ Corresponding author: praphathip.eam@mahidol.ac.th

Received 12 March 2012

Accepted 14 September 2012 immunoblot analysis (Eamsobhana \& Tungtrongchitr 2005, Cross \& Chen 2007, Eamsobhana \& Yong 2009). For some cases, test results fail to provide convincing evidence of the disease because of delays in antibody appearance after infection. In this situation, molecular approaches that target genetic sequences of $A$. cantonensis may assist in providing an aetiologic diagnosis.

In the last decade, various DNA-based diagnostic techniques have become available for detecting nucleic acids of pathogenic parasites in biological materials such as blood, stool, urine, cutaneous scrapings and secretions, including CSF (Tavares et al. 2011). Polymerase chain reaction (PCR) evaluation of CSF has been successfully applied to the diagnosis of a number of CNS infections caused predominantly by parasitic protozoa (Tavares et al. 2011). For helminth parasites, a semi-nested PCR based on the HDP2 DNA sequence has been reported to be specific for the diagnosis of neurocysticercosis caused by larvae of Taenia solium in CSF samples from infected patients (Hernandez et al. 2008).

Recently, the mRNA sequence encoding the $66 \mathrm{kDa}$ native protein from $A$. cantonensis adult worms has been described and used as the basis for developing a PCR protocol for simultaneous unambiguous identification of species of the genus Angiostrongylus. The AC primers are highly specific and have been used successfully to amplify DNA of Angiostrongylus species (Silva et al. 2003, Eamsobhana et al. 2010).

Because of the ease of amplification with high sensitivity and specificity attained by a single-step PCR, the $\mathrm{AC}$ primers directed against the $66-\mathrm{kDa}$ protein gene of 
A. cantonensis could be a potential approach for confirmation of clinical findings of angiostrongyliasis. Here we report the application of this primer set for detection of cerebrospinal fluid (CSF) DNA of $A$. cantonensis from patients diagnosed suspected to have angiostrongyliasis caused by $A$. cantonensis.

From CSF samples submitted to the Parasitology Laboratory, Department of Parasitology, Faculty of Medicine Siriraj Hospital, Bangkok, Thailand, for routine antibody testing (ELISA and/or immunoblot) of tissue-invading parasites, i.e., Gnathostoma spinigerum, A. cantonensis and T. solium metacestodes, 19 CSF specimens were selected for PCR evaluation based on the volume of remaining CSF sample available for DNA analysis. These CSF specimens were from clinically diagnosed cases with positive immunoblot tests for the presence of a 31-kDa band specific for A. cantonensis (n = 10; designated as CSF1-10) and clinically suspected cases with negative immunoblot tests for $A$. cantonensis infection ( $\mathrm{n}=5$; CSF11-15). CSF samples (representing other clinically related parasitic infections) with positive immunoblot tests showing a 24-kDa band specific for $G$. spinigerum $(\mathrm{n}=2$; CSF16-17) and with ELISA-positive cases of $T$. solium neurocysticercosis $(n=2$; CSF18-19) were included in the study. The CSF specimens were maintained at $-80^{\circ} \mathrm{C}$ in the Department of Parasitology at Siriraj Hospital after the initial routine immunological investigations. The use of these remainder clinical CSF samples for this study was approved by the director of Siriraj Hospital, Faculty of Medicine Siriraj Hospital, Mahidol University.

For DNA preparation, $500 \mu \mathrm{L}$ of an individual CSF sample was concentrated to a final volume of $200 \mu \mathrm{L}$ by ultrafiltration with a Centricon Ultracel YM-100 device (Millipore Corporation, Billerica, MA, USA) according to the manufacturer's protocol. The genomic DNA was extracted from each concentrated CSF sample using a proteinase $\mathrm{K}$ digestion step followed by purification of the DNA on silica-gel spin columns (QIAamp DNA mini kit, QIAGEN, Germany). Prior to DNA extraction, $5 \mu \mathrm{g}$ poly dA (Sigma Chemical Co, USA) was added as a DNA carrier to $200 \mu \mathrm{L}$ of AL lysis buffer (provided in the QIAGEN kit), as recommended by the manufacturer. The DNA purification procedure was performed according to the manufacturer's instructions for the extraction of biological fluids.

The presence of $A$. cantonensis DNA was confirmed by using the oligonucleotide primer pairs AC1: 5'CTCGGCTTAATCTTTGCGAC-3' and AC2: 5'AACGAGCGGCAGTAGAAAAA-3' (Silva et al. 2003). The extracted A. cantonensis DNA (Thailand isolate) obtained from our previous investigations (Eamsobhana et al. 2010) was used as a reference positive control in the PCR amplification. The sequences were aligned using the CLUSTALW program (Thompson et al. 1994), as implemented in BioEdit 7.0.1 (Hall 1999), using default parameters.

The positive PCR amplification yielded a fragment of approximately $280 \mathrm{bp}$. PCR products were detected in four (CSF2, 3, 5 and 7) of the $10 \mathrm{CSF}$ samples from patients showing clinical criteria of eosinophilic meningitis

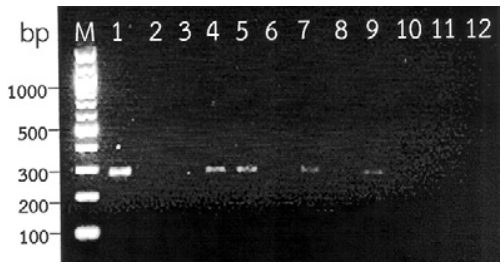

Fig. 1: gel electrophoresis showing positive polymerase chain reaction (PCR) products from cerebrospinal fluid (CSF) samples of four infected patients with serological positive angiostrongyliasis cantonensis (Lane 4: CSF2; 5: CSF3; 7: CSF5; 9: CSF7). Lanes 3 (CSF1), 6 (CSF4), 8 (CSF6), 10 (CSF8), 11 (CSF9) and 12 (CSF10) are negative for amplification products by PCR. Lane 1: positive control; 2: negative control (master mix); M: molecular weight markers (100-bp ladder).

and positive results for a $31-\mathrm{kDa}$ A cantonensis-specific immunoblot band. No amplification occurred from the CSF samples from patients who showed clinical features of infection, but were negative for $A$. cantonensis-specific antibodies. The specificity of the AC primers pair was demonstrated by the absence of the amplification product in the CSF samples from patients with cerebral gnathostomiasis and neurocysticercosis. A typical amplicon pattern of positive and negative results is shown in Fig. 1.

Three of the positive amplicons (CSF2, 3 and 5) yielded good sequencing results. Following alignment, a 250-bp sequence was obtained. A high degree of homology was present among the sequences and only a few deletions and transitions were observed (Fig. 2). The sequences were related to the reference $A$. cantonensis sequence fragment (AC-TF) with 98.8\%, 98.8\% and 99.2\% nucleotide similarity for CSF2, 3 and 5, respectively.

The specific AC primers were previously used to amplify a 232-bp fragment from three patients with histopathologically confirmed abdominal angiostrongyliasis (Silva et al. 2003). In the present study, we have tested clinical CSF samples and found evidence for the presence of $A$. cantonensis DNA in four of 10 patients diagnosed with meningitic angiostrongyliasis. The lack of DNA amplification in the other six patients, despite parameters suggesting a high probability of $A$. cantonensis infection, may represent false negative results. The development of more sensitive PCR-based methods is needed for use with samples from patients in whom the burden of worms/DNA is below the threshold for detection. This study was not designed to measure the clinical sensitivity of PCR as a diagnostic test because complete clinical information on all the subjects was not available. Consequently, additional CSF samples from $A$. cantonensis-infected patients for whom complete clinical data are available should be evaluated.

Based on our results, DNA purification on silica spin columns may be used as a routine method of CSF preparation for PCR analysis. The major constraint on this DNA preparation method is the limited volume of clinical CSF samples usually available after their use for routine investigations. A higher volume of CSF will be required for DNA purification, particularly in cases of infection in which low numbers of worms are present in the CSF. 

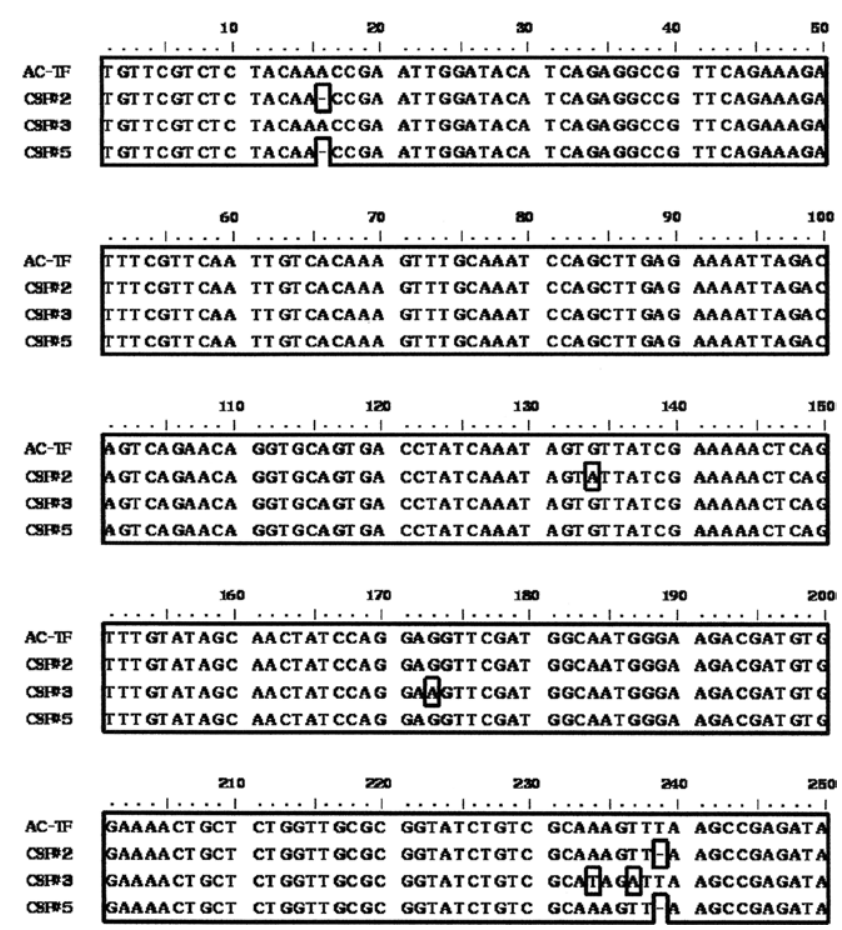

Fig. 2: alignment of the nucleotide sequences of the 66-kDa protein gene of Angiostrongylus cantonensis amplified by polymerase chain reaction (PCR) (primers $\mathrm{AC1}$ and $\mathrm{AC} 2$ ) from positive cerebrospinal fluid (CSF)-PCR products from angiostrongyliasis patients. CSF\#2, CSF\#3 and CSF\#5 are CSF-PCR positive samples from patients; AC-TF is the reference sequence from adult female $A$. cantonensis Thailand isolate.

Studies of the detection of $A$. cantonensis DNA in clinical CSF samples to support a diagnosis of eosinophilic meningitis due to A. cantonensis have been extremely limited. In this study, by using a standard PCR, we demonstrated the presence of $A$. cantonensis DNA in CSF samples from patients with neuroangiostrongyliasis. Although the PCR assay has yet to be assessed with CSF samples from parasitologically proven cases (because no samples from proven CSF cases were available for analysis), the assay was able to detect $A$. cantonensis DNA in CSF samples from patients who were serologically positive for infection.

\section{ACKNOWLEDGEMENTS}

To the director of the Siriraj Hospital, Faculty of Medicine Siriraj Hospital, Mahidol University, for permission to carry out this work, to Nuntiya Monkong and Suphaluck Wankhom, for assistance in retrieval of CSF samples kept at the Department of Parasitology, Siriraj Hospital, to Kanokwan Ngamsuwan and Kornthip Meesuk, for technical assistance, and to the editor and three anonymous reviewers whose remarks and suggestions were very useful in improving the paper.

\section{REFERENCES}

Cross JH, Chen ER 2007. Angiostrongyliasis. In KD Murrel, B Fried, Food borne parasitic zoonoses, Springer, New York, p. 263-290.

Eamsobhana $\mathrm{P}$ 2006. The rat lungworm Parastrongylus (=Angiostrongylus) cantonensis: parasitology, immunology, eosinophilic meningitis, epidemiology and laboratory diagnosis, Wankaew (IQ) Book Center, Bangkok, 156 pp.

Eamsobhana P, Lim PE, Zhang H, Gan XX, Yong HS 2010. Molecular differentiation and phylogenetic relationships of three
Angiostrongylus species and Angiostrongylus cantonensis geographical isolates based on a 66-kDa protein gene of $A$. cantonensis (Nematoda: Angiostrongylidae). Exp Parasitol 126: 564-569.

Eamsobhana P, Tungtrongchitr A 2005. Angiostrongyliasis in Thailand. In N Arizono, JY Chai, Y Nawa, T Takahashi, Food-borne helminthiasis in Asia, The Federation of Asian Parasitologists, Chiba, p. 32-47.

Eamsobhana P, Yong HS 2009. Immunological diagnosis of human angiostrongyliasis due to Angiostrongylus cantonensis (Nematoda: Angiostrongylidae). Int J Infect Dis 13: 425-431.

Graeff-Teixeira C, da Silva ACA, Yoshimura K 2009. Update on eosinophilic meningoencephalitis and its clinical relevance. Clin Microbiol Rev 22: 322-348.

Hall TA 1999. Bioedit: a user-friendly biological sequence alignment editor and analysis program for Window 95/98NT. Nucleic Acids Symp Ser 22: 4673-4680.

Hernandez M, Gonzalez LM, Fleury A, Saenz B, Parkhouse RM, Harrison LJ, Garate T, Sciutto E 2008. Neurocysticercosis: detection of Taenia solium DNA in human cerebrospinal fluid using a seminested PCR based on HDP2. Ann Trop Med Parasitol 102: 317-323.

Silva ACA, Graeff-Teixeira C, Zaha A 2003. Diagnosis of abdominal angiostrongyliasis by PCR from sera of patients. Rev Inst Med Trop Sao Paulo 45: 295-297.

Tavares RG, Staggemeier R, Borges ALP, Rodrigues MT, Castelan LA, Vasconcelos J, Anschau ME, Spalding SM 2011. Molecular techniques for the study and diagnosis of parasite infection. J Venom Anim Toxins Incl Trop Dis 17: 239-248.

Thompson JD, Higgins DG, Gibson TJ 1994. CLUSTALW: improving the sensitivity of progressive multiple sequence alignment through sequence weighting, position specific gap penalties and weight matrix choice. Nucleic Acids Res 22: 425-430.

Wang QP, Lai DH, Zhu XQ, Chen XG, Lun ZR 2008. Human angiostrongyliasis. Lancet Infect Dis 8: 621-630. 